\title{
sur la gestion et les finances publiques En Juillet 2021
}

\section{BUDGET DE L'ÉTAT ET DES OPÉRATEURS}

\section{Données générales sur les finances publiques}

Le PIB se redresse au deuxième trimestre $2021(+0,9 \%)$, et se rapproche de son niveau d'avant-crise

Dans une note parue le 30 juillet 2021, I'INSEE indique que le PIB français se redresse au deuxième trimestre $2021(+0,9 \%)$, après une stabilité au trimestre précédent. Le PIB comble ainsi plus d'un cinquième de l'écart restant fin 2020 par rapport à son niveau d'avant-crise : il est inférieur de 3,3\% au niveau du quatrième trimestre 2019, contre 4,2\% aux deux trimestres précédents.

La demande intérieure finale contribue positivement à l'évolution du PIB ce trimestre $(+0,9$ point, après $+0,1$ point au trimestre précédent). La formation brute de capital fixe accélère ( $+1,1 \%$ après $+0,4 \%$ au premier trimestre) de même que les dépenses de consommation des ménages (+ 0,9\% après + 0,2 \%). L'INSEE précise également que les importations augmentent plus fortement (+1,9\%) que les exportations (+ 1,5\%). Au total, la contribution du commerce extérieur à la croissance du PIB est faiblement négative : $-0,1$ point, après $-0,5$ point au trimestre précédent.

\section{$\pi$ Procédure budgétaire}

La loi du 19 juillet 2021 de finances rectificative pour 2021

La loi de finances rectificative pour 2021 a été promulguée le 19 juillet 2021 et publiée au Journal officiel du 20 juillet 2021.

Ce collectif budgétaire de transition vise principalement à accompagner les entreprises dans la sortie de crise jusqu'à la fin de l'année 2021. II met également en place des mesures de soutien au pouvoir d'achat des salariés et aux plus précaires et contient des dispositions accompagnant la réouverture au public dans les secteurs du sport et de la culture, ainsi que la poursuite du plan de relance. Aussi, le budget rectificatif comprend plusieurs mesures en faveur des collectivités locales, comme un mécanisme de compensation des pertes de recettes de leurs régies industrielles et commerciales. Un amendement du Sénat reconduit pour 2021 le « filet de sécurité » pour certaines ressources spécifiques des collectivités territoriales d'outre-mer et de Corse.

In fine, la prévision de déficit public pour 2021 est revue à $-9,4 \%$ du PIB, avec un taux d'endettement public qui atteindrait 117,2\% du PIB à la fin de l'année. Le solde budgétaire est de - 220 Mds€.

\section{Administrations budgétaires}

Le financement de la recherche publique dans la lutte contre la pandémie de Covid19

Dans le contexte de la crise sanitaire, la Cour a décidé de lancer pour la première fois un " audit flash » pris en application d'une des mesures phare de son rapport « JF2025 ». II a été publié le 29 juillet 2021. Le rapport, tout en respectant les normes professionnelles traditionnelles, se signale par la rapidité de son instruction, réalisée dans un délai de quatre mois. Ce travail se présente comme un premier bilan relatif à l'effort financier consenti en faveur de la recherche publique dans le cadre de la lutte contre la pandémie, sur la période allant du

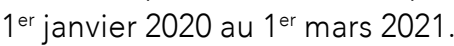

Sur la période qui court de janvier 2020 à mars 2021, le volume total des ressources mises en 
place est de l'ordre de $502 \mathrm{M} €$. Cet effort est moindre, dans l'absolu comme relativement, que celui consenti par d'autres pays européens aux moyens et au tissu scientifique comparables.

Ainsi, en dépit des efforts particulièrement méritoires de tous les acteurs du système de recherche français, et malgré les qualités internationalement reconnues de nos chercheurs, les résultats ne sont pas à la hauteur des attentes. L'éparpillement des initiatives, la difficile mise au point de priorités, l'impréparation à la prise de risque et les lourdeurs administratives ont contrarié la création d'un système de réponse cohérent et efficace.

Au bilan, la Cour estime qu'il a manqué une stratégie de recherche. L'absence de chef de file pour la mettre en œuvre a notamment été pénalisante. Au-delà des évaluations sur les volumes financiers débloqués en situation critique et des observations sur des défauts d'organisation en temps de crise, l'audit met également - voire surtout - en évidence des défauts structurels qu'il recommande de corriger.

\section{$\pi$ Budget des pouvoirs publics}

Les comptes et la gestion des services de la présidence de la République (exercice 2020)

Le 26 juillet 2021, la Cour des comptes a rendu public son rapport annuel relatif au contrôle des comptes et de la gestion des services de la présidence de la République. Le thème du rapport porte cette année sur la sécurité de la Présidence.

La Cour constate que le principe consistant à s'appuyer sur deux forces, d'une part, la Garde républicaine en charge de la défense à la fois des emprises présidentielles et du Président à l'intérieur de celles-ci ; d'autre part, le Groupe de Sécurité de la Présidence de la République (GSPR) protégeant le chef de l'État à l'extérieur, a été réaffirmé. Pour les coordonner, un échelon supérieur a été créé, l'état-major opérationnel, tandis que des mutualisations, en particulier des fonctions de soutien, ont été réalisées. Concomitamment, les relations financières et fonctionnelles avec le ministère de l'intérieur qui met à disposition les personnels en charge de la sécurité ont été clarifiées.

Dans ce cadre, la mise en place d'une nouvelle Direction de la Sécurité de la Présidence de la République (DSPR), constitue une avancée qui permet d'optimiser les ressources en vue d'une plus grande efficacité opérationnelle. Les pre- mières étapes ont été franchies avec succès. La montée en puissance de la direction nécessite à présent d'être consolidée.

A cet égard, La Cour considère que si la DSPR dispose d'une analyse de risques depuis 2020, cette dernière doit encore être complétée pour devenir un outil de pilotage stratégique et d'anticipation. Le rapport préconise qu'afin de poursuivre et renforcer ce travail, une démarche visant à solliciter davantage des regards extérieurs adaptés à la spécificité de la Présidence de la part d'experts en matière de sécurité au sein de l'État pourrait être utilement initiée pour parfaire l'organisation, la formalisation de doctrines consolidées ou l'entraînement commun.

\section{FinANCES LOCALES}

\section{$\pi$ Rapport de la Cour des comptes sur les aides à l'investissement local}

La Cour des comptes vient de rendre un nouveau rapport intitulé Les concours financiers de l'État en soutien à l'investissement public local. Dans un contexte de relance de l'investissement, cette analyse des juges financiers prône la remise en cause de certains aspects. En premier lieu, la Cour des comptes alerte sur l'importance de doter le comptable des moyens de contrôler le FCTVA. En effet, avec I'automatisation de ce dispositif, le contrôle de légalité $n$ 'interviendra plus. En second lieu, les juges attirent l'attention sur le fait que les subventions profitent principalement aux grandes aires urbaines délaissant un certain nombre de territoires. En troisième lieu, la Cour des comptes relève que la démarche de subventions devrait davantage s'inscrire dans une logique de pluriannualité et de performance.

\section{Délibération du Comité des finances locales (CFL) en date du 20 juillet 2021}

Les récentes réformes fiscales (taxe d'habitation et impôts de production) nécessitent une modification des indicateurs mesurant la richesse fiscale et financière. Le Comité des finances locales souhaite une réforme de ceux-ci dès le projet de loi de finances pour 2022, alors que le directeur général des collectivités semblait indiquer qu'aucune réforme ne serait présentée dans le prochain projet de loi de finances. Le CFL affirme sa volonté de lisser dans le temps la mise en œuvre des nouveaux indicateurs. Le CFL propose d'intégrer l'effet des indicateurs dans le cadre des garanties prévues il y 
a deux ans lors de la réforme de la dotation d'intercommunalité. Sur le fond, le CFL souhaite inclure dans le potentiel financier d'autres recettes libres d'affectation non utilisées pour l'instant, comme les DMTO, la taxe locale sur la publicité extérieure, la taxe sur les pylônes électriques ou encore la majoration de la taxe $d$ 'habitation sur les résidences secondaires. En revanche, il refuse d'inclure des recettes affectées comme la taxe de séjour ou la taxe locale sur la consommation finale d'électricité.

Enfin, le Comité des finances locales confirme son accord de principe sur le remplacement du mode de calcul de l'effort fiscal en intégrant le revenu.

\section{$\pi$ Les finances des collectivités locales en 2020}

La direction générale des collectivités locales (DGCL) vient de publier une étude sur les finances des collectivités locales en 2020. L'analyse confirme les tendances déjà observées avec en premier lieu un besoin de financement de 0,5 Mds€ confirmant la dégradation des finances locales sous l'effet de la crise sanitaire.

Dans le détail, parmi les constats les plus significatifs on pourra retenir que la dette des régions, après une quasi-stabilité en 2019, retrouve un taux de progression très fort (+ $9 \%$ ), comparable aux évolutions des années 2014 et 2015. Celle des départements (+ 3,2\%) revient à son niveau de 2018 après avoir reculé quatre années de suite. En comparaison, les mouvements du secteur communal paraissent plus modérés : I'encours de dette progresse de $+1,6 \%(+0,3 \%$ pour les communes et $+5,1 \%$ pour les groupements à fiscalité propre).

L'épargne brute de l'ensemble des collectivités a diminué en 2020 car les recettes de fonctionnement ont diminué $(-1,6 \%)$ tandis que les dépenses ont continué d'augmenter (+ 0,2\%).

Enfin, le financement des investissements communaux par l'épargne brute (c'est-à-dire par l'excédent de la section de fonctionnement) a été plus difficile en 2020. Ce constat ne se limite pas au secteur communal (- 5,5\% sur l'épargne brute) : c'est le cas aussi pour les départements dont l'épargne a chuté (-14,1\%), comme pour les régions (- 21,6\%).

\section{Publication des données de calcul des dotations}

Pour la $4^{\text {e }}$ année de suite, la DGCL publie le détail des fichiers permettant le calcul des dotations par strate de collectivités. Si on peut saluer cette démarche de transparence, notons toutefois que toutes les données ne sont pas publiques. La publication des données permet ainsi de recalculer les dotations pour vérifier d'éventuelles erreurs. Cela permet aussi de comprendre plus précisément l'impact de la population dans le dynamisme de la DGF ou encore de comprendre les redéploiements internes de la DGF.

Notons qu'à ce jour ne sont toujours pas publiées les données sur les régions.

\section{$\lambda$ Rebond probable des dépenses d'in- vestissement en 2021}

Cette étude codirigée par la Banque postale et la Fédération nationale des travaux publics permet d'apporter de premiers éléments sur 2021 et sur l'impact financier de la sortie de crise. Cette analyse repose sur une enquête adressée à un certain nombre de collectivités. II apparaît que $67 \%$ des répondants envisagent une hausse des dépenses d'investissement. Pour 2022, les collectivités n'ont pas encore rendu tous les arbitrages et à ce stade, une stabilité des dépenses se profile.

En matière de travaux publics, la voirie et la transition écologique occupent le haut du pavé démontrant ainsi progressivement les mutations de la société. Il s'agit également d'une conséquence du plan de relance. Les collectivités ont à cet égard largement eu recours au plan de relance et en matière d'emprunts elles recourent davantage aux prêts « verts ».

Notons toutefois que les inégalités régionales sont réelles. Parmi les régions dans lesquelles I'investissement sera fortement en hausse en 2021 on trouverait l'île-de-France, la Nouvelle-Aquitaine et le Centre-Val de Loire. À I'inverse, dans les Hauts-de-France la hausse apparaît plus incertaine.

\section{$\pi$ Discussion du projet de loi 3DS au Sénat}

Les travaux parlementaires au Sénat de la loi 3DS ont modifié plusieurs dispositions en matière financière. La première concerne les modalités d'attribution de la dotation de soutien à l'investissement local (DSIL). Le Sénat souhaite que la décision relève du préfet du département au lieu du préfet de région. Les sénateurs aimeraient également que l'affectation de la DSIL s'effectue par l'intermédiaire d'une commission départementale composée d'élus locaux. En matière de compensation financière 
liées aux transferts de compétences, le Sénat a introduit une révision tous les cinq ans pour « les coûts d'exercice et de gestion » mais également en matière d'inflation. L'une des dernières dispositions concerne le futur contrat, si contrat il y a, entre l'État et les collectivités. La Haute Assemblée a souhaité introduire une disposition selon laquelle "les dépenses de solidarité sociale des collectivités territoriales fixées par la loi sont exclues de tout objectif national visant à encadrer l'évolution des dépenses réelles de fonctionnement des collectivités territoriales et de leurs groupements à fiscalité propre ».

\section{FINANCES SOCIALES}

\section{तituation des comptes sociaux et conséquences de la crise sanitaire}

Les annonces du Président de la République sur la poursuite des réformes

Au-delà de ses annonces sur les mesures pour faire face à la 4 e vague de l'épidémie (généralisation du "passe sanitaire», vaccination obligatoire des soignants, conditions de l'isolement) le Président de la République s'est exprimé le 12 juillet sur la suite des grandes réformes dans le domaine social. S'agissant de la réforme des retraites, il a confirmé le report de l'âge de départ, tout en tenant compte de la difficulté des métiers, ainsi que la garantie d'une pension d'au moins $1000 €$ pour une carrière complète, mais il a subordonné son lancement à la mise sous contrôle de l'épidémie et à l'affermissement de la reprise économique, le Premier ministre devant lancer une nouvelle concertation à la rentrée. En direction des jeunes, après avoir rappelé les résultats du programme " 1 jeune, 1 solution » qui a bénéficié à 2 millions d'entre eux, il a annoncé, pour la rentrée, la mise en œuvre d'un "Revenu d'engagement», avec des obligations de formation ou de recherche d'emploi, qui pourrait être une extension de la «Garantie Jeunes», un comité de 18 personnalités ayant été désigné pour poursuivre la réflexion engagée à ce sujet depuis le début de l'année. Pour les personnes âgées, il a annoncé une grande ambition pour l'autonomie et le maintien à domicile. Enfin, il a confirmé la pleine mise en œuvre de la réforme de l'Assurance chômage le 1er octobre prochain, avec le lancement d'un plan massif de formation et de requalification des chômeurs de longue durée. Ces réformes devront être financées sans hausse d'impôt ni dérapage excessif du déficit.
La Cour des Comptes dresse un bilan des aides à l'emploi pour faire face à la crise sanitaire

Dans son rapport sur l'action du ministère du travail et de l'emploi pour le soutien des entreprises face à la crise sanitaire, la Cour rappelle tout d'abord l'ampleur des moyens mis en œuvre (31,8 Md€ en 2020 et 29,8 Md€ en 2021, en $\mathrm{CP}$ toutes missions budgétaire confondues), et met en lumière la profusion des dispositifs : activité partielle, mesures du plan « 1 jeune, 1 solution », aides aux entreprises sociales inclusives, prolongation des droits des chômeurs, " année blanche " des intermittents du spectacle, soutiens à Pôle Emploi et France Compétence... Elle relève que l'objectif initial de préservation de l'emploi a été atteint, mais que les résultats ont pu être inégaux selon les dispositifs, et surtout que le coût de certains d'entre eux n'a pas été maîtrisé (formations non qualifiantes, prolongation des droits des chômeurs de longue durée, année blanche très généreuse pour les intermittents...). Elle insiste surtout sur le fait que les mesures de contrôle ont été trop tardives et trop insuffisamment ciblées (la référence étant les dispositifs anti-fraude mis en œuvre par les URSSAF et la DGFiP), et formule 10 recommandations à cet effet.

\section{$\lambda$ Dépenses de santé /Assurance maladie/hôpital}

Le rapport d'information de l'Assemblée Nationale sur les Agences régionales de santé

La mission d'évaluation et de contrôle des lois de financement de la Sécurité sociale de l'Assemblée Nationale a déposé un rapport d'information sur les ARS. Elle part d'abord du constat que 10 ans après leur création par la loi HPST en avril 2010, celles-ci n'ont pas encore trouvé leur place dans le paysage administratif et que leurs insuffisances se sont particulièrement révélées lors de la crise sanitaire : leur périmètre de compétence, qui a eu l'objectif de regrouper l'action des ARH, des Unions régionales des caisses de Sécurité sociale (URCAM), des groupements régionaux de santé publique et d'une partie des compétences des DDASS et des Caisses régionales d'Assurance maladie s'est révélé trop large pour être bien appréhendé, si bien que les ARS se sont focalisées sur I'offre de santé - et essentiellement celle de l'hôpital au détriment de la politique de santé publique (prévention et sécurité sanitaire)-, même si elles ont pu approfondir leur relation avec le secteur médico-social; (leur situation mal définie par 
rapport aux préfets et l'existence d'une double chaîne de commandement ont beaucoup compliqué la gestion de la crise de la COVID 19 à ses débuts); I'ampleur du territoire qu'elles couvrent au niveau régional les a éloignées des problématiques de terrain, les délégations départementales des ARS n'ayant qu'une compétence résiduelle; leur statut ambigu, unique au sein des administrations publiques du fait du copilotage des politiques de santé entre l'État et l'Assurance maladie en fait un « objet administratif non identifié ». La mission d'évaluation formule 23 propositions qui s'articulent autour de 3 axes : 1) renforcer la légitimité des ARS, particulièrement en renouant avec la proximité et en renforçant le partenariat avec les préfets, et aussi avec les élus locaux au niveau du département ; 2) mettre la subsidiarité au cœur de l'exercice de leur mission, notamment en clarifiant celle-ci dans la gestion des crises, et en privilégiant le contrôle a posteriori des établissements de santé ; 3) revoir le pilotage de la dépense de santé, dans le sens d'une plus grande régionalisation, sans pour autant aller jusqu'à la régionalisation de l'ONDAM.

\section{Le rapport " charges/produits " de la CNAM pour 2022}

Comme chaque année à la même époque, la CNAM publie son rapport « charges/produits» qui contient des propositions pour améliorer la qualité du service de santé et maîtriser les dépenses. Cette année, la CNAM consacre de larges développements à la crise de la COVID 19, qui a abouti à un déficit abyssal de $30 \mathrm{Md€}$ de l'Assurance maladie en 2020. La CNAM fait un ensemble de 36 propositions : 11 d'entre elles visent, pour 2022, à réaliser $1 \mathrm{Md}$ d'économies ; 25 propositions concernent plutôt le moyen terme, dans la perspective de repenser largement la stratégie du régime et ses modalités de conception, de pilotage et d'évaluation pour prendre en compte le contexte nouveau dans lequel s'inscrivent les politiques de santé.

\section{Le bilan du "Ségur de la Santé ", un an après son lancement}

Le ministère des solidarités et de la santé a publié le 20 juillet un dossier de presse pour faire le bilan du «Ségur de la Santé » lancé le 13 juillet 2020 par le Premier ministre. Ce plan a comporté tout particulièrement un programme de $9 \mathrm{Md} €$ pour la revalorisation des métiers. Ces revalorisations, qui englobent le "socle» de $183 €$ nets mensuels accordés à tous les personnels et des révisions des grilles indiciaires propres à chaque catégorie de soignants et de personnel médical et non médical, sont détaillées dans le dossier : par exemple un infirmier de soins généraux aura bénéficié après 5 ans de carrière d'une augmentation de $335 €$ nets par mois, un aide-soignant de $368 €$ (du fait du passage en catégorie $\mathrm{B}$ ), un praticien hospitalier de 1 104€ nets par mois (du fait des mesures pour renforcer l'attractivité des carrières hospitalo-universitaires)....Le Ségur de la Santé comporte également de nombreuses mesures pour le fonctionnement de I'hôpital (aménagement du temps de travail), ainsi qu'un plan de $19 \mathrm{Md}$ d'investissements dans le système de santé, dont 15,5 Md€ pour le volet sanitaire, 2,3 Md€ pour le médico-social et 1,4 Md€ pour le numérique. Par ailleurs, dans la continuité de la stratégie " Ma santé 2022 » lancée en 2018, il accélère la réduction de la part de la tarification à l'activité (T2A) dans le financement des établissements et confirme l'engagement des travaux de réforme de l'ONDAM suite à l'avis du Haut Conseil pour l'avenir de l'Assurance maladie d'avril 2021 ainsi que la poursuite de la transformation du système de santé dans les territoires.

Les complémentaires santé : d'un rapport très mitigé de la Cour des Comptes à un projet de «Grande Sécu » ?

La Cour des Comptes a rendu public le 22 juillet le rapport qu'elle a établi à la demande de I'Assemblée Nationale sur les complémentaires santé. La Cour fait tout d'abord le constat que la France n'a pas choisi un système de plafonnement annuel des dépenses de santé par ménage (dispositif du "bouclier sanitaire »), mais a préféré limiter les restes à charge par divers dispositifs d'exonération (ex. ALD) et favoriser le développement des complémentaires santé. Des dispositifs spécifiques ont été progressivement instaurés pour aider les ménages aux plus faibles revenus qui ne pouvaient recourir aux assurances privées, avec la CMU-C, puis I'Assurance complémentaire santé (ACS), et enfin la Complémentaire santé solidaire (CSS). II s'ensuit que le dispositif français est très protecteur puisque le niveau du reste à charge est le plus faible des pays de l'OCDE (6,9\%). Mais il apparaît très coûteux pour les finances publiques -10 Md€ par an - (y compris les dépenses fiscales et sociales pour l'incitation à la souscription des complémentaires privées) et en forte progression, même s'il est partiellement couvert par la taxe de solidarité additionnelle, avec une maîtrise très difficile des coûts des organismes du fait de leur morcellement. Par ailleurs, il n'at- 
teint qu'imparfaitement l'objectif de réduction de la renonciation volontaire aux soins et il est plus favorable aux salariés du privé qu'à ceux du public. La Cour propose diverses pistes, fondées sur un meilleur partage des rôles entre les régimes de base et les complémentaires : mise en place d'un plafonnement du reste à charge par rapport aux revenus des assurés par un panier de soins pris en charge par l'Assurance maladie obligatoire, désimbrication des domaines d'intervention respectifs des régimes obligatoires et complémentaires, mise en concurrence accrue des organismes, meilleur encadrement des frais de gestion. Par ailleurs, le ministre des solidarités et de la santé vient de saisir le Haut conseil pour l'avenir de l'Assurance maladie (HCAAM) pour étudier la faisabilité d'une nouvelle articulation entre les complémentaires santé et l'assurance maladie obligatoire, ces propositions devant être rendues pour fin novembre; le HCAAM avait rendu au début de I'année un rapport avec 4 scénarios d'évolution (voir Repères de janvier 2021), dont le plus radical était l'intégration de tout ou partie des garanties en matière de maladie dans l'assurance maladie obligatoire («Grande Sécu »).

\section{$\pi$ Retraites}

Le rapport d'information de la Commission des affaires sociales du Sénat sur les réserves des régimes de retraites

La mission d'évaluation et de contrôle de la Sécurité sociale et la Commission des Affaires sociales du Sénat ont déposé un rapport d'information sur les réserves des régimes de retraite. Elle part du constat d'une situation très contrastée des réserves des régimes avant la crise sanitaire : à fin 2019, 98 \% des réserves du système de retraite (179 Md€ de situation patrimoniale nette) étaient détenues par les régimes complémentaires ; par ailleurs, le Fonds de réserve des retraites n'apparaît pas en mesure de remplir l'objectif qui lui avait été fixé à sa création en 1999, à savoir assurer le financement de la « bosse démographique du papy-boom », du fait des ponctions qu'il a subies à partir de 2011 pour renflouer la CADES. Avec la survenue de la crise sanitaire, la situation patrimoniale nette des régimes de retraite s'est réduite en 2020 de près de $18 \mathrm{Md}$, notamment du fait de la poursuite jusqu'en 2033 des versements du FRR à la CADES. La mission du Sénat recommande donc de limiter à l'avenir l'usage des réserves des caisses de retraite à la seule préservation de l'équilibre financier des régimes qu'elles gèrent pour faire face aux chocs économiques, sans tentation de mobiliser celles-ci pour la solidarité inter-régimes.

\section{Le huitième avis du Comité de suivi des retraites}

Le Comité de suivi des retraites institué par la loi du 20 janvier 2014 rend un avis annuel au regard des 4 objectifs que doit remplir le système de retraites : verser des pensions en rapport avec les revenus d'activité ; traiter équitablement les assurés; assurer la solidarité intra et inter générationnelle; préserver la pérennité financière. Le $8^{e}$ avis du CSR du 15 juillet 2020 (soit 6 mois seulement après le précédent qui avait été fortement décalé à cause de la crise) prend en compte les hypothèses économiques, démographiques et financières du COR de juin dernier (voir Repères de juin) et fait le constat que les prévisions, exprimées en termes de soldes financiers, sont moins mauvaises que celles produites à l'automne 2020, du fait de la reprise escomptée de la croissance en 2021 ; mais il émet des réserves sur la lisibilité de ces indicateurs de solde et les messages qu'ils envoient, du fait des conventions comptables du COR qui sont conditionnées par l'effort budgétaire de l'État pour financer les régimes de retraite publics. Le CSR propose de raisonner à partir du ratio dépenses de retraites/PIB, en se basant sur un taux un peu inférieur au niveau actuel hors effet de la crise de la COVID, soit $13,5 \%$ du PIB et, de là, il envisage 2 possibilités : 1) considérer que le niveau de $13,5 \%$ est pertinent, et opérer l'ajustement, sans effort financier supplémentaire, par un report de l'âge de départ à 64 ans et accepter une baisse de l'ordre de $10 \%$ du revenu des retraités ; 2) considérer que le niveau de $13,5 \%$ est trop élevé, au regard des besoins de financement des autres besoins collectifs de la nation, ce qui impliquerait un recul plus marqué de l'âge de départ et /ou un ajustement à la baisse plus important du niveau de vie des pensionnés, avec la nécessité de se montrer prudent sur le recul de l'âge d'ouverture des droits, compte-tenu de la situation du marché du travail qui reporterait alors le déficit sur l'Assurance chômage, et de la tendance à la stabilisation de l'espérance de vie.

\section{L'accord sur les mesures de redressement} de I'AGIRC-ARRCO

Selon les informations parues dans Les Echos, pour faire face au déficit de I'AGIRC-ARRCO (voir Repères de juin), le patronat et deux syndicats (CFDT et CFTC) se sont mis d'accord pour autoriser le Conseil d'administration à sous-in- 
dexer (jusqu'à 0,5 points) la revalorisation des pensions par rapport à l'inflation en 2021 et 2022 ; les trois autres syndicats (CGT, CFE-CGC et $\mathrm{FO}$ ) auraient préféré une ponction sur le montant des réserves.

\section{$\lambda$ Dépenses liées à la famille/politique du logement}

L'accord entre les Pouvoirs publics et Action Logement sur l'évolution du groupe

La ministre du logement et les partenaires sociaux d'Action Logement ont publié le 19 juillet un communiqué commun formalisant leur accord sur les évolutions à apporter à la stratégie et la gouvernance du groupe. Un rapport de I'Inspection générale des finances, au début de 2020, avait fortement critiqué la politique d'Action Logement (qui gère la participation des employeurs à l'effort de construction - ou PEEC, ex $1 \%$ logement - qui s'est élevée à 1,35 Md€ en 2020), organisme qui a pour mission de construire et financer des logements sociaux et intermédiaires, ainsi que d'accompagner les salariés dans leur mobilité résidentielle et professionnelle. L'accord précise l'implication d'Action Logement dans le plan de relance et confirme les évolutions que le groupe doit mettre en œuvre dans trois directions : le renforcement de sa structuration pour une meilleure efficacité ; une plus grande ouverture avec la création d'un comité des partenaires et d'un comité de déontologie; I'amélioration de la gouvernance avec l'instauration d'un comité d'orientation politique, ces évolutions devant s'inscrire dans une réflexion sur le modèle économique en vue de la future convention quinquennale. Le gouvernement s'est par ailleurs engagé à ne pas transférer aux URSSAF ou à la DGFiP la collecte de la PEEC, et à ne pas effectuer de prélèvement sur les réserves de l'organisme dans le PLF 2022, comme cela avait été souvent le cas dans le passé.

Le premier bilan de la réforme des aides publiques au logement

Le ministère de la transition écologique a publié le 22 juillet un premier bilan de la très controversée réforme des APL qui est entrée en vigueur au $1^{\text {er }}$ janvier 2021 et qui a visé à calculer les APL sur la base des revenus des 12 derniers mois, au lieu de prendre en compte ceux de l'année N-2, sans autre modification des paramètres de calcul. Selon le ministère, la réforme a conduit, sur les près de 6 millions d'allocataires, à diminuer l'allocation de janvier 2021 pour 29,6 \% d'entre eux, à l'augmenter pour $18,2 \%$ et à la maintenir à l'identique pour $52,2 \%$. L'économie réalisée sera finalement supérieure à ce qu'escomptait le gouvernement, soit 1,1 Md€ de moins qu'en 2020 (contre 075 Md€ prévus en LFI 2021.)

\section{$\lambda$ Assurance chômage}

\section{Les comptes 2020 de I'UNEDIC certifiés} avec réserves

Pour la première fois, les commissaires aux comptes de I'UNEDIC ont certifié avec 2 réserves les comptes du régime d'Assurance chômage. Cela tient à l'importance des mesures d'urgence mises en place pour faire face à la crise sanitaire, qui n'ont pas permis de mettre en œuvre les contrôles internes sur I'information financière produite par deux opérateurs: I'ACOSS, tête de réseau des URSSAF qui assurent le recouvrement des cotisations chômage, s'est trouvée dans la situation d'impossibilité de certification de ses comptes par la Cour des Comptes (voir Repères de mai) ; l'Agence des services de paiement (ASP) qui verse les allocations de chômage partiel en liaison avec les Directions régionales de l'économie, de l'emploi, du travail et des solidarités (DREETS) ne fait pas l'objet d'un processus de certification. Les CAC ont toutefois émis une opinion favorable pour l'activité d'indemnisation des demandeurs d'emploi et pour la gestion financière du régime.

\section{FISCALITÉ ET PROCÉDURES FISCALES}

\section{$\lambda$ Politique fiscale}

Loi de finances rectificative pour 2021 et ses mesures fiscales

Le Sénat a adopté, le 1er juillet, avec modifications, par 247 voix contre 28, le projet de loi de finances rectificative pour 2021 (v. "Repères", mai 2021). Parmi les 25 articles qui étaient soumis au Sénat, 10 articles ont été adoptés conformes et 15 dispositions ont été modifiées. Le Sénat a également introduit 26 nouveaux articles. Finalement, 41 articles du projet de loi sont donc restés en discussion en CMP. Au plan fiscal, il prévoit : I'aménagement temporaire du dispositif de report en arrière des déficits « carry back» (art. 1 - CGl, art 220 quinquies) ; la mise en conformité avec le droit de l'UE et la jurisprudence du Conseil d'État (CE, 14 oct. 2020, AVM Holding International, Req. $n^{\circ}$ 421524) du prélèvement applicable aux gains résultant 
de la cession ou du rachat de droits sociaux ainsi qu'à certaines distributions réalisées soit par une personne physique non domiciliée en France, soit par une personne morale dont le siège social est situé hors de France (art. 2 CGI, art. 244 bis B) ; la prolongation jusqu'au 31 décembre 2021 de la déductibilité des abandons de créances de loyers consentis par les bailleurs (art. 8 - CGI, art. 14B et 39-1-9); la majoration exceptionnelle du taux de la réduction d'impôt sur le revenu au titre des dons effectués au profit des associations cultuelles (art. 18 - CGI, art 200) ; la prorogation jusqu'au 31 décembre 2022 de la hausse de $18 \%$ à $25 \%$ du taux de la réduction à l'impôt sur le revenu pour la souscription au capital des PME et ETI («IR-PME »- art. 19) ; I'application aux discothèques fermées administrativement depuis plus d'un an d'un dégrèvement systématique de taxe foncière jusqu'à une date fixée par décret (art. 21 - CGl, art. 1639 A bis). En CMP, les parlementaires ont en définitive rejeté l'amendement des sénateurs visant à réactiver le dispositif d'exonération des dons familiaux dans la limite de $100000 €$ qui a pris fin au 30 juin dernier (CGl, art. 790 A bis). La loi n ${ }^{\circ}$ 2021-953 du 19 juillet 2021 de finances rectificative pour 2021 a été publiée au JORF du 20 juillet 2021. Elle n'a pas fait l'objet d'une saisine devant le Conseil constitutionnel.

La stabilité de la législation fiscale dans le cadre d'une LOLF modifiée pour ses 20 ans?

Les députés ont voté une proposition de loi organique modifiant la gouvernance des finances publiques portée par le rapporteur général du budget et le président de la commission des finances de l'Assemblée nationale. Depuis trois ans, les parlementaires des commissions des finances (v. rapport MILOLF 2019 à I'A.N. ; groupe de travail "LOLF » mis en place au Sénat) travaillent à la modernisation de la loi organique relative aux lois de finance (LOLF), dont on va fêter cette année les 20 ans. Plusieurs dispositions de ce texte concernent la fiscalité. Il faut noter un renforcement de la doctrine d'affectation des taxes affectées à des tiers autres que les collectivités territoriales et les organismes de sécurité sociale (art. 3); une clarification des exigences applicables aux prélèvements sur recettes (art. 4) ; la création d'une nouvelle catégorie des lois de finances, la « loi de fin de gestion » au sein desquelles serait exclu l'ensemble du domaine facultatif des lois de finances, en particulier toute disposition relative aux ressources de l'État et à la fiscalité affectée, une exception étant prévue pour les dispositions tendant à modifier pour l'année en cours l'affectation d'impositions de toute nature (art. 6). Le texte adopté par les députés, le 20 juillet, a été renvoyé à la commission des finances du Sénat pour un examen au cours de la session extraordinaire de septembre. À suivre...

\section{入 Impôt sur le capital}

ISF : après le feuilleton judiciaire « Finaréa " devant la Cour de cassation, ses suites

Les suites du feuilleton judiciaire (v. Repères, janv. et mars 2021) de l'affaire "Finaréa »? Dernièrement, la Cour de cassation a rendu une nouvelle décision dans le cadre d'un contentieux ISF qui confirme que l'effectivité du caractère animateur d'une Holding implique de réunir des éléments factuels attestant ce rôle d'animation. Selon la Cour de cassation, le caractère effectif du rôle d'animation de la société holding au sein du groupe doit être caractérisé concernant les conventions d'animation, les procès-verbaux du conseil de surveillance de la société Holding, et les rapports annuels de gestion présentés par le directoire au conseil de surveillance de la société Holding (Cass. Com., 23 juin 2021, Société Holding E., pourvoi n 1916.351). Cette décision révèle la difficulté qu'il y a à se prévaloir du caractère animateur d'une holding sans éléments patents. À suivre...

Le rendement budgétaire de l'impôt sur la fortune immobilière (IFI) en 2020

L'IFI a rapporté 1,56 Mds€ en 2020, soit 500 M€ de moins qu'en 2019 en incluant les sommes dues au titre de I'IFI des années précédentes et de l'ancien ISF; ou en progression de 4,4\% au regard du seul IFI qui a eu un rendement budgétaire de 1,49 Mds€ en 2019. Selon la note publiée par la DGFIP en juillet, il est possible d'observer la grande hétérogénéité des contribuables assujettis à I'IFI : un quart seulement a un patrimoine immobilier supérieur à 2,5 M€. Selon cette note, plus de 143000 foyers ont adressé en 2020 une déclaration d'IFI, en progression de $3 \%$ sur un an.

\section{Contrôle fiscal}

\section{Rapport d'activité de Tracfin}

Le rapport annuel d'activité de Tracfin pour l'année 2020 démontre une hausse du nombre de déclarations de soupçon émises auprès d'elle (112 962) de $18 \%$ par rapport à 2019 après avoir reçu 115601 informations. En dépit du contexte 
sanitaire, Tracfin a poursuivi ses missions prioritaires. Les banques et établissements de crédit demeurent les premiers contributeurs avec 62033 signalements effectués (+ $10 \%$ ) pour un enjeu financier total de 23,9 Mds€. En 2020, Tracfin a transmis 3033 notes d'information et de renseignement à l'autorité judiciaire et à I'administration fiscale. Le rapport souligne I'efficacité du dispositif français de la lutte anti-blanchiment (v. «Repères », juillet 2020).

\section{$\lambda$ Impôt sur les sociétés}

Accord sur de nouvelles règles internationales concernant la fiscalité des entreprises

Après le sommet du G7 du 5 juin 2021, 130 États de I'OCDE (90\% du PIB mondial) sont parvenus le 10 juillet 2021 à un accord sur une réforme de la taxation des multinationales, prévoyant notamment la mise en place d'un impôt minimum sur les bénéfices d'au moins $15 \%$. Ces États ont adhéré à la Déclaration qui instaure un nouveau cadre pour la réforme de la fiscalité internationale. Les éléments restants du cadre de la réforme, y compris le plan de mise en $œ u v r e$, seront finalisés en octobre 2021, pour une mise en œuvre effective en 2023. Le directeur de la fiscalité à l'OCDE Pascal Saint-Amans pense que cet accord signe «la fin des paradis fiscaux ». Même la Suisse y a adhéré, "sous certaines conditions ». La Commission européenne s'est félicitée de cette avancée du droit international fiscal.

Le cadre de référence établi repose sur deux piliers. II vise à faire en sorte que les grandes entreprises multinationales paient des impôts là où elles exercent des activités et génèrent des bénéfices, tout en renforçant la sécurité juridique et la stabilité du système fiscal international. Le premier pilier garantit une répartition plus équitable des bénéfices et des droits d'imposition entre États concernant les grandes entreprises multinationales, y compris celles du numérique. Il permettra de réattribuer une partie des droits d'imposition sur les entreprises multinationales de leurs États d'origine aux États de marché dans lesquels elles exercent des activités commerciales et réalisent des bénéfices, qu'elles y aient ou non une présence physique. Au titre du premier pilier, des droits d'imposition sur plus de 100 Mds \$ de bénéfices devraient être réattribués chaque année aux juridictions de marché. Le second pilier entend encadrer la concurrence fiscale en matière d'impôt sur les bénéfices des sociétés en introdui- sant un impôt minimum mondial que les États peuvent prélever pour protéger leur base d'imposition. Avec un taux d'au moins $15 \%$, l'impôt minimum mondial sur les bénéfices des sociétés au titre du second pilier devrait générer environ 150 Mds\$ de recettes fiscales supplémentaires par an au niveau mondial.

La note du Conseil des prélèvements obligatoires sur le futur impôt mondial

Le Conseil des prélèvements obligatoires (CPO) considère, dans une note intitulée "Les enjeux pour la France des négociations à l'OCDE sur la taxation des bénéfices des multinationales », publiée le 8 juillet, que la réforme fiscale internationale va apporter des recettes supplémentaires (les recettes liées au pilier $n^{\circ} 2$ serait de I'ordre de $5 \mathrm{Mds} €$ ), et qu'il est clairement dans I'intérêt de la France de signer cet accord multilatéral au regard des pertes de recettes fiscales observées depuis 2008. Il lance toutefois un avertissement sur les risques lors de sa mise en $œ u v r e$, notamment sur la définition de l'assiette taxable. Ces craintes ont été aussi exprimées par la doctrine (v. «Repères », juin 2021).

\section{La France continue à prélever la taxe GAFA jusqu'en 2023}

L'administration fiscale a annoncé continuer à prélever la taxe GAFA jusqu'en 2023. Celle-ci ne disparaîtrait que lorsque la grande réforme de l'impôt mondial conduite par l'OCDE serait effective. Le ministre de l'économie et des finances s'est dit «prêt à prendre un engagement formel vis-à-vis des États-Unis » sur la disparition de la taxe. Dans l'attente, le calendrier de versement des acomptes de la taxe GAFA demeure inchangé cette année.

Acte anormal de gestion : exigence de détail suffisant pour la proposition de rectification

La juridiction administrative vient de rappeler dans le cadre d'un acte anormal de gestion que la proposition de rectification doit être suffisamment détaillée et chiffrée en "éléments servant de base au calcul de l'impôt " quant au montant des rehaussements envisagés. II résulte des dispositions législatives et règlementaires (LPF, art. L. 57 et R. 57-1) que l'administration fiscale doit indiquer au contribuable, dans la proposition de rectification, les motifs et le montant des rehaussements envisagés, leur fondement légal et la catégorie de revenus dans laquelle ils sont opérés, ainsi que les années d'imposition concernées, de manière à permettre au contri- 
buable de formuler ses observations de façon entièrement utile (CAA Paris, 6 juillet 2021, $n^{\circ}$ 19PA02380).

\section{Management PUblic}

\section{$\pi$ Relations avec les usagers}

Le dispositif de traitement des signalements de violence et de discrimination à Matignon

Un arrêté du 19 juillet 2021 fixe le dispositif de recueil et de traitement des signalements d'actes de violence à caractère sexuel, de discrimination, de harcèlement sexuel et d'agissements sexistes dans les services du Premier ministre. "Ce dispositif est ouvert à l'ensemble des agents des services du Premier ministre qui s'estiment victimes ou témoins d'actes de violence à caractère sexuel, de discrimination, de harcèlement sexuel ou d'agissements sexistes sur leur lieu de travail ou dans l'exercice de leurs fonctions. II est également ouvert aux agents ayant quitté les services du Premier ministre depuis moins de six mois, ainsi qu'aux candidats à un recrutement dont la procédure a pris fin depuis trois mois au plus» (article 1).

Cet arrêté a été pris en application du décret du 13 mars 2020 qui avait précisé le contenu des cellules de signalement que chaque administration doit mettre en place, conformément à la loi de transformation de la fonction publique du 6 août 2019.

\section{$\pi$ Fonction publique}

\section{Les derniers concours de I'ENA}

89 places sont offertes aux concours 2021 de l'École nationale d'administration (ENA), contre 83 l'année dernière, selon un arrêté du 20 juillet 2021. Pour rappel, il s'agit des derniers concours que l'ENA organisera puisqu'elle sera

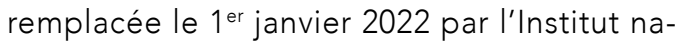
tional du service public (INSP). La hausse du nombre de places s'explique par le concours externe dit "Talents » réservé aux personnes d'origine modeste. En revanche, les nombres de places offertes pour les autres concours de I'ENA restent les mêmes qu'en 2020 : 40 pour le concours externe, 32 pour le concours interne, 7 pour le troisième concours et 4 pour le concours réservé aux docteurs.

\section{EUROPE}

\section{$\pi$ Politique européenne}

Principe du pollueur-payeur : une application européenne incohérente

Dans un rapport spécial $n^{\circ} 12 / 2021$ remis le 5 juillet, la Cour des comptes européenne a présenté son analyse de l'application du principe pollueur-payeur (PPP). Son titre est très clair : « une application incohérente dans les différentes politiques et actions environnementales de I'UE ». La Cour constate ainsi que si le PPP est pris en compte dans plusieurs politiques européennes, le budget de l'UE est parfois utilisé pour financer des actions de dépollution dont les coûts auraient dû, en vertu de ce même PPP, être supportés par les pollueurs. Résultat selon la Cour: "Le contribuable européen paie trop souvent la note à la place du pollueur ». Elle propose plusieurs corrections pour éviter cela.

Journal de la Cour des comptes consacré à la PAC

Le Journal de la Cour des comptes européennes publié le 15 juillet 2021 est entièrement consacré, suite à l'accord interinstitutionnel du 25 juin, à l'analyse prospective de la «nouvelle» Politique agricole commune (PAC), qui demeure un des premiers postes budgétaires européens et doit être durable, équitable et neutre pour le climat.

Lutte contre le blanchiment de capitaux et le financement du terrorisme

Le 20 juillet 2021, la Commission a présenté un ensemble considéré comme ambitieux de propositions législatives visant à renforcer les règles de l'UE en matière de lutte contre le blanchiment de capitaux et le financement du terrorisme.

\section{Aides d'État : simplification des règles}

Le 23 juillet, la Commission européenne a adopté une proposition d'extension du champ d'application du règlement général d'exemption par catégorie (RGEC) qui permettrait de simplifier les règles applicables pour aider à la double transition et à la reprise. Si elle a poursuivi en ce mois de juillet une application très compréhensive du contrôle des aides d'État, il faut toutefois noter que la Commission a ouvert le 26 juillet une enquête approfondie concernant la rémunération versée par la FDJ à la France. 


\section{Pacte de stabilité}

Conclusions du Conseil sur les bilans approfondis de 2021

Le 13 juillet 2021, le Conseil ECOFIN a publié ses conclusions sur les bilans approfondis de 2021 dans le cadre de la procédure concernant les déséquilibres macroéconomiques. En substance, le Conseil considère " qu'il est important de poursuivre la coordination étroite des politiques économiques de I'UE, y compris de détecter, prévenir et corriger les déséquilibres macroéconomiques qui entravent le bon fonctionnement des économies des États membres, de l'Union économique et monétaire ou de l'économie de l'Union européenne dans son ensemble ». II partage l'appréciation de la Commission selon laquelle la crise de la COVID-19 n'a pas fondamentalement changé la nature des déséquilibres des États membres, mais a entrainé un recul dans la réduction des déséquilibres observée précédemment et pourrait accroître les risques pesant sur la stabilité macroéconomique, notamment le niveau des dettes souveraines. Et constate " qu'il est encore difficile de prendre toute la mesure des conséquences de la crise, y compris ses effets structurels ».

\section{$\pi$ Budget européen}

Conditionnalité liée à l'État de droit : le Parlement européen souhaite des enquêtes

Dans une résolution adoptée le 8 juillet par 529 voix pour, 150 contre et 14 abstentions, les députés regrettent que la Commission ait décidé de se conformer aux conclusions non contraignantes du Conseil européen de décembre 2020 et de retarder I'application du règlement sur la conditionnalité pour la protection du budget de l'Union en élaborant des lignes directrices relatives à son application.

Feu vert aux fonds de I'UE relatifs à la reprise

Les 13 juillet et 28 juillet 2021, le Conseil ECOFIN a donné son feu vert aux premiers décaissements nationaux dans le cadre du plan de relance européen, pour respectivement 12 puis 4 États membres.

Position du Conseil sur le budget européen 2022 et budget rectificatif 2021

Le 14 juillet, les ambassadeurs auprès de I'UE ont approuvé la position du Conseil sur le pro- jet de budget de I'UE pour 2022. Au total, la position du Conseil prévoit 167,7 Mds€ en crédits d'engagement et $170 \mathrm{Mds} €$ en crédits de paiement. Le budget pour 2022 sera complété par des fonds de Next Generation EU destinés à contribuer à stimuler la reprise économique européenne. Rappelons qu'au budget rectificatif 2021, notamment en raison de droits de douanes plus élevés que prévus et d'une sous-exécution des dépenses, avait été inscrit l'excédent de l'exercice 2020, correspondant à $1768617610 €$, ce qui avait suscité des débats au Parlement européen.

\section{$\lambda$ Euro}

\section{La BCE maintient son soutien à l'économie}

Le 22 juillet 2021, si la Banque centrale européenne (BCE) s'est inquiétée des conséquences économiques du variant Delta, elle a annoncé le maintien de son soutien à l'économie, en fixant un objectif d'inflation proche mais inférieur à $2 \%$ qui lui laisse le temps de durcir sa politique monétaire dont les taux directeurs sont toujours fixés entre 0 et $0,5 \%$.

\section{入 Fiscalité}

Pandémie : exonération temporaire de TVA sur les " achats et dons "

Le 13 juillet, le Conseil ECOFIN a adopté une modification de la directive TVA introduisant une exonération temporaire de la TVA sur les importations et certaines opérations réalisées en réponse à la pandémie de COVID-19. La directive dite «d'achat et de don» permettra à la Commission et aux agences de l'UE d'acheter plus facilement des biens et des services pour les distribuer gratuitement aux États membres dans le contexte de la crise de santé publique actuelle.

\section{INTERNATIONAL}

\section{त États}

\section{La Federal Reserve reste confiante face à} l'inflation

Le 15 juillet, le président de la Réserve fédérale J. Powell a annoncé maintenir sa politique monétaire. S'il y a des raisons de s'inquiéter face au renforcement de l'inflation $(3,4 \%$ estimés cette année), il estime que cela devrait se stabiliser à moyen terme avec un taux de $2,1 \%$ en 2022 et 2,2 \% en 2023. 


\section{$\pi$ Fiscalité}

Conséquence de l'accord au G20 en faveur d'un taux minimal d'IS sur les GAFA

Dans la foulée des accords du G20 sur la fiscalité des multinationales (cf. rubrique "Fiscalité et procédure fiscale "), I'administration américaine a demandé à l'UE d'abandonner son projet de taxe sur les GAFA, pourtant prévue pour financer le remboursement du plan de relance. Le gel de cette taxe, annoncé dès le 12 juillet, ne simplifie pas le dossier des ressources propres européennes. D'autant que Mme Yellen, la Secrétaire d'État au Trésor, estime que Amazon ne sera sans doute pas soumise totalement à la future taxe, faute de dégager dans toutes ses activités segmentées le surprofit de $10 \%$ qui en sera la condition d'application.

Aurélien BAUDU (Fiscalité et procédure fiscale),

Fabrice BIN (Europe, international),

Florent GAULLIER-CAMUS (budget de l'État et des opérateurs),

Matthieu HOUSER (finances locales), Christophe SINNASSAMY (management public),

Yves TERRASSE (finances sociales) Robin DEGRON (coordination).

Retrouvez sur www.rgfp.fr :

- I'intégralité des REPÈRES d'actualité des finances et de la gestion publiques depuis 2015 - le 15 du mois, les REPÈRES d'actualité du mois précédent. 